\title{
Huygens Excitation in Debye Media in the FDTD Method
}

DOI:

10.1109/TAP.2016.2569198

\section{Document Version}

Accepted author manuscript

Link to publication record in Manchester Research Explorer

\section{Citation for published version (APA):}

Abdulkareem, B., Costen, F., Himeno, R., \& Yokota, H. (2016). Huygens Excitation in Debye Media in the FDTD Method. IEEE Transactions on Antennas and Propagation, 64(8), 3632-3635.

https://doi.org/10.1109/TAP.2016.2569198

\section{Published in:}

IEEE Transactions on Antennas and Propagation

\section{Citing this paper}

Please note that where the full-text provided on Manchester Research Explorer is the Author Accepted Manuscript or Proof version this may differ from the final Published version. If citing, it is advised that you check and use the publisher's definitive version.

\section{General rights}

Copyright and moral rights for the publications made accessible in the Research Explorer are retained by the authors and/or other copyright owners and it is a condition of accessing publications that users recognise and abide by the legal requirements associated with these rights.

\section{Takedown policy}

If you believe that this document breaches copyright please refer to the University of Manchester's Takedown Procedures [http://man.ac.uk/04Y6Bo] or contact uml.scholarlycommunications@manchester.ac.uk providing relevant details, so we can investigate your claim.

\section{OPEN ACCESS}




\section{Huygens Excitation in Debye Media in the FDTD Method}

\author{
Buraq Abdulkareem, Student Member, IEEE, Jean-Pierre \\ Bérenger, Fellow IEEE, Fumie Costen, Senior \\ Member, IEEE, Ryutaro Himeno, Hideo Yokota,
}

\begin{abstract}
The total-field/scattered-field method, also called the Huygens surface method, is a common method to enforce an incident wave in a FDTD grid filled with a vacuum. In this paper it is shown that it can also be used in such dispersive media as the Debye media. The incident wave to be enforced on the Huygens surface is computed either by an analytical calculation in the general case or by means of the auxiliary grid technique in special cases. As in a vacuum, the leakage of energy outside the Huygens surface is lower than $-40 \mathrm{~dB}$ with the analytical incident wave and it can be far lower with the auxiliary grid incident wave.
\end{abstract}

Index Terms-Huygens surface, total field/scattered field, FDTD, dispersive media, biomedical engineering, auxiliary grid.

\section{INTRODUCTION}

Electromagnetic simulators have become an essential tool with applications ranging from telecommunications to radar systems and design of high speed electronic circuit boards as well as health-care devices in biomedical engineering. Such applications need to address the behaviour of physical electromagnetic waves in the time domain during propagation in a wide range of media such as a dispersive human body. There are several approaches to solve Maxwell equations numerically. Among them, the Finite Difference Time Domain (FDTD) method has become the most widely used [1], capable of explicitly computing macroscopic transient electromagnetic interactions with general three dimensional geometries. A fine spatial sampling, strong absorbing boundary conditions, and appropriate source excitations contribute to its high accuracy.

One of the commonly used techniques to excite the FDTD space is the Huygens surface method, otherwise called the total field/scattered field (TF/SF) method [1]. Relying on the equivalence theorem, it permits physical sources to be replaced with fictitious ones. This is necessary when the physical sources are outside the FDTD domain, but can also be used in other situations [2]. A drawback to the TF/SF method derives from the FDTD dispersion. As the frequency approaches the cutoff frequency of the grid, there is a growing leakage of energy into the scattered field region, where the field should be zero. To overcome this problem, several methods have been proposed over the years to implement equivalent sources that match the dispersion of the grid. Tan and Potter [3] developed

B. Abdulkareem, J.-P. Bérenger, F. Costen are with the School of Electrical and Electronic Engineering, The University of Manchester, U.K. (email: fumie.costen@manchester.ac.uk)

R. Himeno is Advanced Center for Computing and Communication, RIKEN, Saitama, Japan

H. Yokota and F. Costen are with the Image Processing Research Team, Center for Advanced Photonics, RIKEN, Saitama, Japan.

Color versions of the figures in this paper are available online at http://ieeexplore.ieee.org. a 1-D multi-point auxiliary propagator for 2-D simulations so as to match the dispersion, in 2-D, at specific angles to the 1-D dispersion. In [4] they proposed the FDTD Discrete Plane wave formulation, where leakage error is at the level of the computer precision at any angle and frequency range, using six 1-D FDTD auxiliary grids.

Beside such sophisticated implementations in a vacuum, use of the TF/SF technique has been also reported in lossy [5] and anisotropic [6] layered media. It has also been used in frequency dependant Debye media with the subgridding technique [7][8], which relies on Huygens surfaces for the connection of grids. The present paper addresses a more classical use of the TF/SF method in Debye media, namely when it is used to generate a known incident wave. Numerical experiments are reported to assess the method and to evaluate its effectiveness. The initial motivation of the work was the need of a tool for exciting plane waves in Debye media, allowing experiments in such bioelectromagnetic areas as in the current work on the extension of the absorbing boundary condition [9] to Debye media. The TF/SF method may also be useful in the future to simulate EM propagation from sources such as electronic devices which transmit information and diagnostics inside the human body.

Section II of the paper briefly describes the implementation of the Huygens surface in the Debye media where the FDTD solution relies on use of an auxiliary equation to compute the electric $\boldsymbol{E}$ field as a function of the displacement $\boldsymbol{D}$ field at each FDTD step. Section III shows one dimensional (1D) and three dimensional (3D) experiments. The reported results show that the wave generated inside the Huygens surface matches the incident wave and that the leakage in the scattered field region remains small.

\section{The Huygens Source In Debye Media}

\section{A. The Huygens source in the FDTD scheme}

The scheme used in this paper is the one in [7][8]. It relies on the auxiliary equation obtained by discretising the time domain counterpart of the equation $\boldsymbol{D}(\omega)=\epsilon_{r}(\omega) \epsilon_{0} \boldsymbol{E}(\omega)$ where the relative permittivity in the Debye medium $\epsilon_{r}$ reads

$$
\epsilon_{r}(\omega)=\epsilon_{\infty}+\frac{\epsilon_{\mathrm{S}}-\epsilon_{\infty}}{1+\jmath \omega \tau}+\frac{\sigma}{\jmath \epsilon_{0} \omega}
$$

where $\epsilon_{\mathrm{S}}$ and $\epsilon_{\infty}$ are the relative static and optical permittivities, $\sigma$ is the conductivity, and $\tau$ is the relaxation time. One time step consists of using the Maxwell-Ampere equation to advance the $\boldsymbol{H}$ field, as in a vacuum, the Maxwell-Faraday equation to advance the $\boldsymbol{D}$ field, and finally the auxiliary equation to obtain $\boldsymbol{E}$ as a function of the advanced $\boldsymbol{D}$.

Let us consider the 1D Huygens surface in Fig. 11. The nodes from $H_{z}\left(i_{1}+\frac{1}{2}\right)$ to $E_{y}\left(i_{2}\right)$ are in the total field region while the nodes up to $E_{y}\left(i_{1}\right)$ and from $H_{z}\left(i_{2}+\frac{1}{2}\right)$ are in the scattered field region. To advance $H_{z}\left(i_{1}+\frac{1}{2}\right)$, the incident electric field $E_{\text {inc } 1}$ at node $i_{1}$ must be added to $E_{y}\left(i_{1}\right)$ in the space derivative, which gives a FDTD equation,

$$
\begin{array}{r}
H_{z}^{n+\frac{1}{2}}\left(i_{1}+\frac{1}{2}\right)=H_{z}^{n-\frac{1}{2}}\left(i_{1}+\frac{1}{2}\right) \\
-\frac{\Delta t}{\mu_{0} \Delta x}\left[E_{y}^{n}\left(i_{1}+1\right)-E_{y}^{n}\left(i_{1}\right)-E_{i n c 1}^{n}\right] .
\end{array}
$$




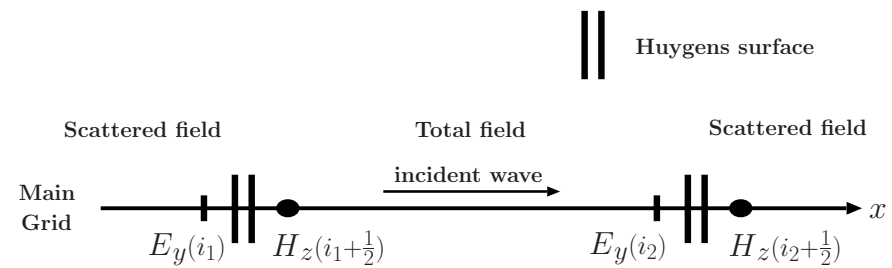

Fig. 1. The Huygens surface in the 1D FDTD grid. Displacement $D_{y}(i)$ is computed at the same nodes as field $E_{y}(i)$.

Similarly, to advance $D_{y}\left(i_{1}\right)$ the incident field $H_{\text {inc1 }}$ must be subtracted to $H_{z}\left(i_{1}+\frac{1}{2}\right)$ which yields

$$
\begin{array}{r}
D_{y}^{n+1}\left(i_{1}\right)=D_{y}^{n}\left(i_{1}\right)-\frac{\Delta t}{\Delta x}\left[H_{z}^{n+\frac{1}{2}}\left(i_{1}+\frac{1}{2}\right)\right. \\
\left.-H_{i n c 1}^{n+\frac{1}{2}}-H_{z}^{n+\frac{1}{2}}\left(i_{1}-\frac{1}{2}\right)\right] .
\end{array}
$$

The component $E_{y}\left(i_{1}\right)$ is then obtained from $D_{y}\left(i_{1}\right)$ using the auxiliary equation given in [10]. Similar equations are used to advance $H_{z}\left(i_{2}+\frac{1}{2}\right)$ and $D_{y}\left(i_{2}\right)$. Notice that the FDTD equations in a vacuum can be viewed as a special case of (2) and (3) where $\boldsymbol{E}$ is obtained with just $\boldsymbol{D}=\epsilon_{0} \boldsymbol{E}$ in place of the auxiliary equation.

\section{B. Production of the incident field}

In a vacuum, the calculation of the incident field, which is the field in the medium if the source were present, is easy. In a Debye medium the calculation is a little more complicated because the medium is lossy and dispersive. Let us consider an incident plane wave propagating to the right, and let us assume, without loss of generality, that we know $E_{i n c 1}$ at node $i_{1}$ of the grid (Fig. 11). To enforce the Huygens surface we must use consistent values of $H_{i n c 1}, H_{i n c 2}$, and $E_{i n c 2}$, at nodes $H_{z}\left(i_{1}+\frac{1}{2}\right), H_{z}\left(i_{2}-\frac{1}{2}\right), E_{y}\left(i_{2}\right)$ respectively. This means that $H_{\text {inc1 } 1}, H_{\text {inc } 2}$ and $E_{\text {inc } 2}$ must be the components of the field that experienced the propagation in the medium from node $E_{y}\left(i_{1}\right)$ to the other three nodes. This can be computed using the relative permittivity (1). Consider for instance $H_{\text {inc1 } 1}$. Taking account of the differences in time, $\Delta t / 2$, and in space, $\Delta x / 2$, it can be expressed [11] in frequency domain as

$$
H_{\text {inc1 } 1}(\omega)=E_{\text {inc1 }}(\omega) \cdot T_{H}(\delta, \omega)
$$

with

$$
\begin{array}{r}
T_{H}(\delta, \omega)=\sqrt{\epsilon_{r}(\omega)} \sqrt{\frac{\epsilon_{0}}{\mu_{0}}} \cdot e^{\jmath \omega\left[\Delta t / 2-\Re\left[\sqrt{\epsilon_{r}(\omega)}\right] \delta / c\right]} \\
\cdot e^{-\omega\left|\Im\left[\sqrt{\epsilon_{r}(\omega)}\right]\right| \delta / c},
\end{array}
$$

where $c$ is the speed of light, $\Re$ and $\Im$ mean the real and imaginary parts of a complex number and $\delta=\Delta x / 2$. From this, $H_{\text {inc1 } 1}(t)$ can be obtained by multiplying the Fourier transform of $E_{i n c 1}(t)$ with the filter $T_{H}$, and by performing an inverse Fourier transform. This reads

$$
H_{\text {inc1 } 1}(t)=\mathcal{F}^{-1}\left[\mathcal{F}\left(E_{\text {inc } 1}(t)\right) \cdot T_{H}(\delta, \omega)\right] .
$$

The Fourier transforms can be easily performed numerically.
Similarly, $E_{i n c 2}$ can be written as

$$
E_{\text {inc2 } 2}(\omega)=E_{\text {inc1 }}(\omega) \cdot T_{E}(\delta, \omega)
$$

with

$$
T_{E}(\delta, \omega)=e^{-j \omega\left[\Re\left[\sqrt{\epsilon_{r}(\omega)}\right] \delta / c\right]} \cdot e^{-\omega\left|\Im\left[\sqrt{\epsilon_{r}(\omega)}\right]\right| \delta / c}
$$

where $\delta=\left(i_{2}-i_{1}\right) \Delta x$, from which

$$
E_{\text {inc } 2}(t)=\mathcal{F}^{-1}\left[\mathcal{F}\left(E_{\text {inc } 1}(t)\right) \cdot T_{E}(\delta, \omega)\right] .
$$

Finally, $H_{\text {inc2 }}$ can be obtained using (5) and (6), with just $\delta=\left(i_{2}+\frac{1}{2}-i_{1}\right) \Delta x$ in place of $\Delta x / 2$.

The Huygens surface can be generalized to the $2 \mathrm{D}$ or $3 \mathrm{D}$ cases in a trivial manner for a plane wave propagating at any incidence angle with respect to the grid. The FDTD nodes of the surface can be placed at the same locations as in a vacuum [1]. Assuming then that the $E$ field is known on a reference plane perpendicular to the propagation of the incident wave, the $E_{i n c}$ and $H_{i n c}$ components can be computed using (6) and (9), with $\delta$ in (5) and (8) equal to the distance from the considered node to the reference plane.

In addition to this analytical calculation, the incident field can also be obtained by means of the auxiliary grid method [1][3][4][5]. In 1D it consists of propagating the incident wave on an auxiliary grid filled with the same medium and discretized with the same steps $\Delta x$ and $\Delta t$. Using as $E_{i n c}$ and $H_{i n c}$ the fields observed at the same locations in the auxiliary grid, the Huygens surface is in theory perfect, without leakage. This is because the incident field has experienced the same dispersion as that of the principal grid. In practice there is a small leakage which is produced by rounding errors, which then depends on the encoding and on the computer. However, the method is rigorous only in $1 \mathrm{D}, 2 \mathrm{D}$, or $3 \mathrm{D}$ when the direction of propagation of the incident wave is parallel to one of the axes of coordinates. Approximate auxiliary grids have been developed for oblique incidence in a vacuum [1][3]. In this paper the method is only used in 1D and in 3D when the propagation is in $x$ direction.

\section{NUMERICAL EXPERIMENTS}

\section{A. ID FDTD space}

1) Simulation set-up: The 1-D FDTD space and time are sampled with $\Delta x=1 \mathrm{~mm}$ and $\Delta t=2 \mathrm{ps}$. The function $100 \exp \left[-\left(\frac{t-125 \Delta t}{25 \Delta t}\right)^{2}\right]$ is used for the electric incident field $E_{\text {inc } 1}$ to correct $E_{y}\left(i_{1}\right)$ in Fig. 1. The entire FDTD space is filled with a Debye medium.

The calculations reported in this paper have been performed with a Skin medium which is one of the most absorbing human tissues. Its one-pole Debye media parameters are $\epsilon_{\mathrm{S}}=47.93$, $\epsilon_{\infty}=29.85, \tau=43.6 \mathrm{ps}, \sigma=0.541 \mathrm{~S} / \mathrm{m}$.

2) Field in the total field region: The width of the total field region in Fig. 1 is 100 FDTD cells. Fig. 2 presents the generated electric field $E_{y}$ at $i=i_{1}+1$, and $i=i_{2}-1$. The incident field enforced on the Huygens surface was computed analytically using (4) - (9). The results are compared with reference fields computed using an auxiliary grid where the 


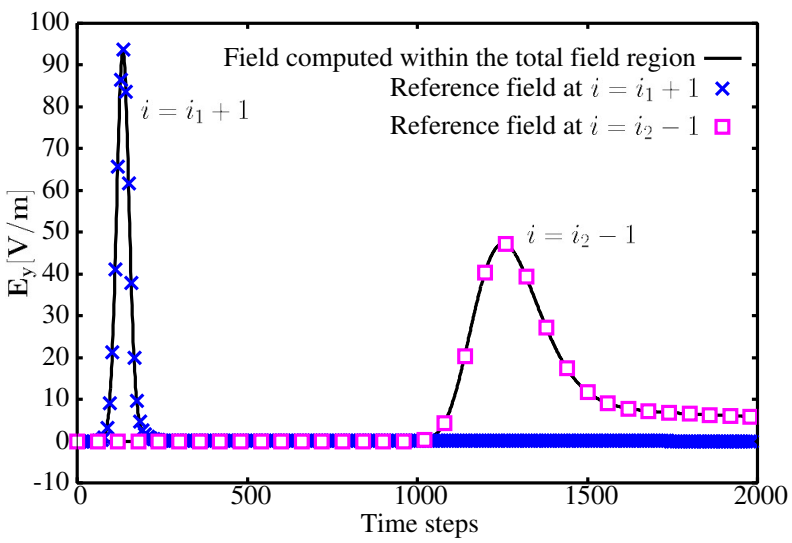

Fig. 2. Total fields for the $1 \mathrm{D}$ experiments. The result at $i_{2}-1$ is multiplied with 10.

incident field was enforced as a hard source at node $E_{y}\left(i_{1}\right)$. A good agreement is observed at the two nodes. The relative errors are quantified by computing the differences between the computed results and the reference solutions as

$$
\varepsilon=100 \frac{\sqrt{\sum_{n}\left|E_{y}(i, n)-E_{y R E F}(i, n)\right|^{2}}}{\sqrt{\sum_{n}\left|E_{y R E F}(i, n)\right|^{2}}}
$$

where $i$ is the node location, either $i_{1}+1$ or $i_{2}-1$, and $n$ is the time step. Formula 10 yields errors $0.37 \%$ at $i_{1}+1$ and $0.67 \%$ at $i_{2}-1$. As expected, the relative error grows with the distance from the left hand side of the Huygens surface since the effect of dispersion grows. However, because the medium is highly absorbing, the absolute error decreases as the wave propagates inside the Huygens surface.

As was discussed in Section II-B, the auxiliary grid method permits the field enforced by the Huygens surface to perfectly match the dispersion of the FDTD grid. In principle, in the total field region the generated fields should be identical to the reference fields computed in the auxiliary grid. In practice, there is a small difference between the two fields, due to rounding errors. Using $(10)$, we obtained errors $0.0022 \%$ at $i=i_{1}+1$ and $0.015 \%$ at $i=i_{2}-1$. These errors are implementation dependant, but in any case they are far lower than the errors obtained with the analytical incident wave.

3) Leakage from the Huygens surface: This is observed at $E_{y}\left(i_{1}-1\right)$ and $E_{y}\left(i_{2}+1\right)$. Fig. 3 shows the leakage obtained when using the analytical incident wave. The peak of the leakage at $i=i_{1}-1$ is $-53 \mathrm{~dB}$ as compared to the peak of the closest node in the interior of the surface and -53.5 $\mathrm{dB}$ as compared to the peak of the incident wave. In contrast at $i=i_{2}+1$ the leakages are $-43.9 \mathrm{~dB}$ and $-70.4 \mathrm{~dB}$. Using the auxiliary grid incident field the observed leakage is far smaller. In theory it is zero. In practice the left surface has a leakage of $-112.3 \mathrm{~dB}$ as compared to the incident field. Similarly, the leakage of the right surface is $-112.7 \mathrm{~dB}$.

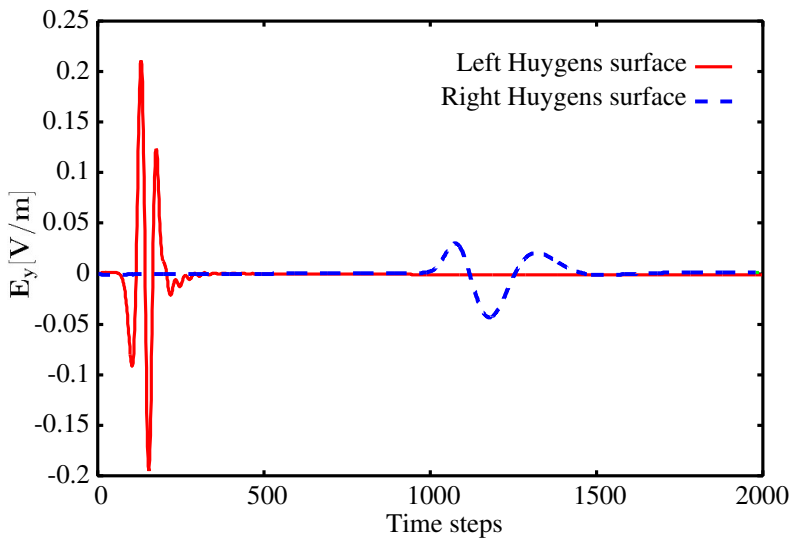

Fig. 3. Scattered field (leakage) in 1D, at two nodes one cell from the left and right hand sides of the Huygens surface.

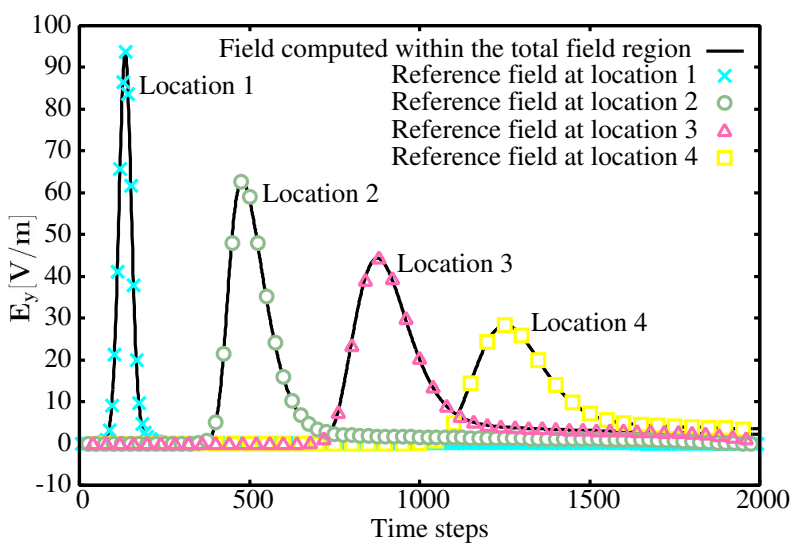

Fig. 4. Total fields for the $3 \mathrm{D}$ experiments. The results at locations 2,3 , and 4 are multiplied by 3,5 , and 6 respectively.

\section{B. 3D FDTD space}

1) Simulation set-up: The 3-D FDTD space is uniformly sampled with $\Delta x=\Delta y=\Delta z=1 \mathrm{~mm}$ and $\Delta t=2 \mathrm{ps}$. The grid size of the 3 -D FDTD space is $200 \times 200 \times 200$ which includes the $100 \times 100 \times 100$-grid Huygens excitation surface. The wave propagates towards the $+x$ direction in the 3-D FDTD space and the Huygens surface is set at the same nodes as in a vacuum [1]. The left hand surface perpendicular to $x$ is set between the plane with index $i=i_{1}-\frac{1}{2}$, which holds $H_{y}$ and $H_{z}$ nodes, and the plane with index $i=i_{1}$, which holds $E_{y}$ and $E_{z}$ nodes. Notice this disposal is the dual of the one used in 1D in Fig. 1. Obviously, setting the surface between $H_{z}\left(i_{1}-\frac{1}{2}\right)$ and $E_{y}\left(i_{1}\right)$ is also possible in 1D. The right hand surface perpendicular to $x$ is set between indexes $i=i_{2}$ and $i=i_{2}+\frac{1}{2}$. The locations of the surfaces perpendicular to $y$ and $z$ are similar and can be obtained by a circular permutation of the coordinates. The same Gaussian function as the one used in 1D is utilised as the electric incident field, with the same analytical calculations (4) - 97. As in 1D, the 3D FDTD space is filled with Skin tissue.

2) Field in the total field region: The $E_{y}$ component parallel to the incident electric field is plotted in Fig. 4 at four locations, two close to the left and right hand sides, namely $E_{y}\left(i_{1}+1, j_{1}+50+\frac{1}{2}, k_{1}+50\right)$ 


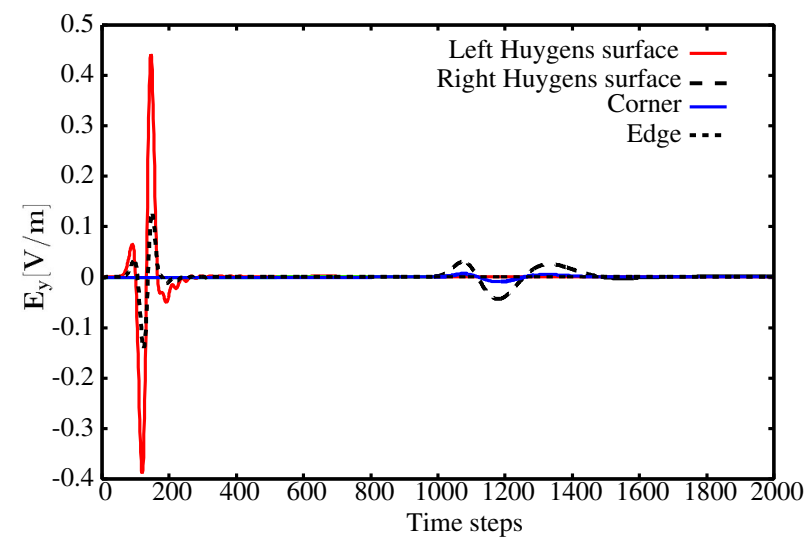

Fig. 5. Scattered field (leakage) in 3D, at four nodes one cell outside the Huygens surface.

and $E_{y}\left(i_{2}-1, j_{1}+50+\frac{1}{2}, k_{1}+50\right)$, and two close to the edges, namely $E_{y}\left(i_{1}+33, j_{1}+3+\frac{1}{2}, k_{1}+3\right)$ and $E_{y}\left(i_{2}-33, j_{2}-3-\frac{1}{2}, k_{2}-3\right)$. The fields at these nodes, denoted as locations 1, 4, 2, 3 in Fig. 4, are compared with references solutions computed as before using a 1D auxiliary grid. As in 1D in Fig. 2, we observe a good agreement. Especially, this means that the Huygens surface planes perpendicular to $y$ and $z$ do not produce a significant spurious field in the total field region. By applying $(10)$ to the four locations, errors $0.51 \%, 0.627 \%, 1.88 \%, 1.58 \%$ are obtained.

3) Leakage: The $E_{y}$ component is represented in Fig. 5at four nodes; all outside the Huygens surface. One node was to the left of the surface, $E_{y}\left(i_{1}-1, j_{1}+50+\frac{1}{2}, k_{1}+50\right)$, one to the right, $E_{y}\left(i_{2}+1, j_{1}+50+\frac{1}{2}, k_{1}+50\right)$, one near an edge, $E_{y}\left(i_{1}-1, j_{2}-50+\frac{1}{2}, k_{1}-1\right)$, and one near a corner, $E_{y}\left(i_{2}+1, j_{2}+1+\frac{1}{2}, k_{2}+1\right)$. Compared to the field at the closest nodes in the interior of the surface, the leakages are $-46.5 \mathrm{~dB},-43.8 \mathrm{~dB},-57.3 \mathrm{~dB}$, and $-57.0 \mathrm{~dB}$ respectively. As compared to the incident wave, they are $-47.1 \mathrm{~dB},-70.4$ $\mathrm{dB},-57.8 \mathrm{~dB}$, and $-83.5 \mathrm{~dB}$. In theory the non-excited components, in our case $E_{x}, E_{z}, H_{x}$, and $H_{y}$, have zero values whereas in practice these components are non-zero. The non-excited components, near the Huygens surface where the largest values are observed, are of the same order as the leakage of the excited components. In particular, components $E_{x}$ and $E_{z}$ are of the same order as the $E_{y}$ leakage plotted in Fig. 5 .

\section{3D FDTD space with oblique incident wave}

The FDTD space is $100 \times 100 \times 100$ which includes a $50 \times 50 \times 50$ Huygens cube. The Gaussain pulse used in Sections III-A, III-B is utilised to generate the excitation. The incident fields are computed using the analytical method. The direction of propagation is perpendicular to $x$ axis and $45^{\circ}$ from $y$ and $z$ axes. Fig. 6 shows the $E_{z}$ component on $i=i_{1}+2$ plane at $t=0.6,1,1.6 \mathrm{~ns}$. The wave is well generated in the total field region with leakage similar to the one observed in Fig. 5 We can observe in Fig. 6 that there is a small residual field behind the pulse, i.e. the color is slightly different from that of the exterior of the Huygens cube. This

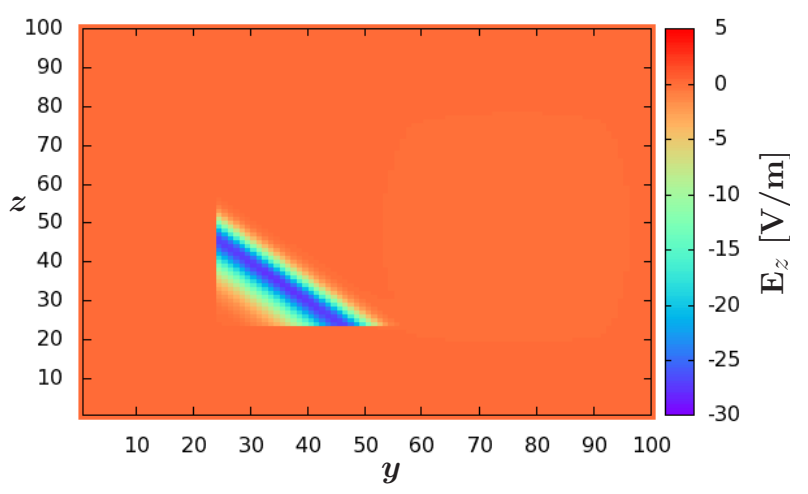

(a) $t=0.6 \mathrm{~ns}$.

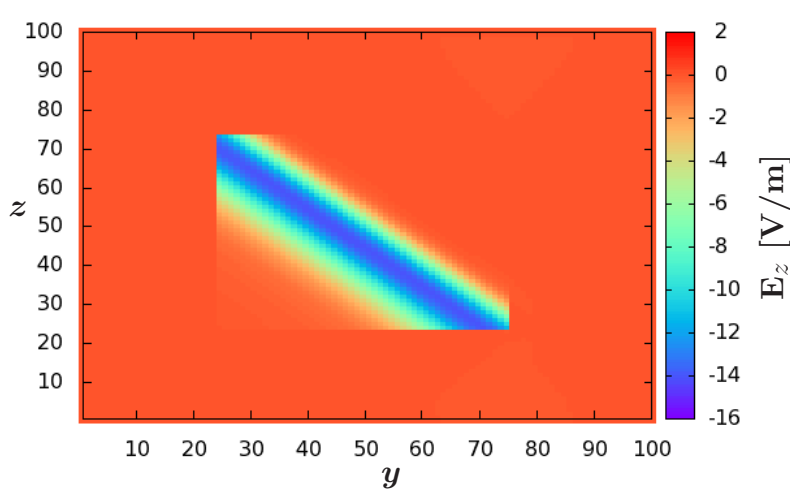

(b) $t=1 \mathrm{~ns}$.

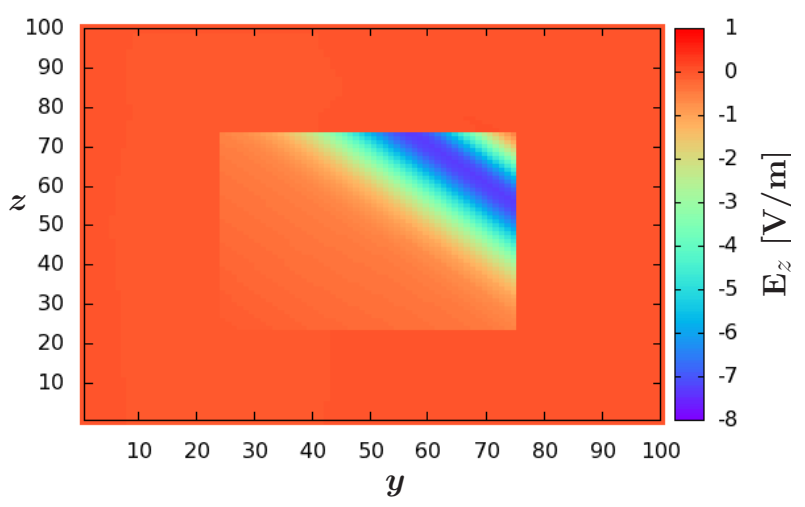

(c) $t=1.6 \mathrm{~ns}$.

Fig. 6. Wave propagating in skin tissue perpendicularly to the $x$ axis and $45^{\circ}$ from the $y$ and $z$ axes.

is because the attenuation of the high frequencies is higher than the one of the low frequencies in Debye media. In other words the pulse is filtered by the Debye medium. This results in the presence of the tail that is also visible in the 1D and 3 D results in Fig. 2 and Fig. 4

\section{CONCLUSION}

The total field/scattered field method can be used to generate an incident wave in the dispersive Debye media. This offers the possibility of placing radiating sources in the human body, whose tissues are well represented by Debye models. Numerical experiments with an incident plane wave have shown that the quality of the generated wave is close to that in a vacuum. In particular, the leakage out of the Huygens surface is less than $-40 \mathrm{~dB}$ when the incident field is enforced as 
an analytical function. Obviously the quality can be far better when the incident wave matches the grid dispersion, which can be easily realized by using an auxiliary grid when the incident field propagates in the direction of one axis of coordinates. A further work could be the extension of the Schneider method [12] to reduce the leakage at oblique incidence. This method could be used with little additional cost since it relies on the dispersive delay of propagation in the FDTD grid, which could be incorporated into the filters $(5)$ and (8).

\section{REFERENCES}

[1] A. Taflove and S. Hagness. Computational Electromagnetics: The finitedifference Time-domain method. Artech House, Boston, MA, 2005.

[2] J.-P. Bérenger. FDTD computation of VLF-LF propagation in the EarthIonosphere Waveguide. Annals of Telecom, vol. 57, pp. 1057-10, 2002.

[3] T. Tan and M. Potter. 1-D Multipoint Auxiliary Source Propagator for the Total-Field/Scattered-Field FDTD Formulation. IEEE Antennas and Wireless Propag. Lett., vol. 6, pp. 144-148, 2007.

[4] T. Tan and M. Potter. FDTD Discrete Planewave (FDTD-DPW) Formulation for a Perfectly Matched Source in TFSF Simulations. IEEE Trans. Antennas Propag., vol. 58, no. 8, pp. 2641-2648, 2010.

[5] I. R. Çapoğlu and G. S. Smith. A Total-Field/Scattered-Field PlaneWave Source for the FDTD Analysis of Layered Media. IEEE Trans. Antennas Propag., vol. 56, no. 1, pp. 158-169, 2008.

[6] Y. Fang, L. Wu, and J. Zhang. Excitation of Plane Waves for FDTD Analysis of Anisotropic Layered Media. IEEE Antennas and Wireless Propag. Lett., vol. 8, pp. 414-417, 2009.

[7] F. Costen and J.-P. Bérenger. Extension of the FDTD Huygens subgridding to frequency dependent media. Ann. Telecommun., vol. 65, pp. 211-217, 2009.

[8] M. Abalenkovs, F. Costen, J.-P. Bérenger, R. Himeno, H. Yokota, and M. Fujii. Huygens Subgridding for 3-D Frequency Dependent FiniteDifference Time-Domain Method. IEEE Trans. Antennas Propag., vol. 60, no. 9, pp. 4336-4344, 2012.

[9] J.-P Bérenger. On the Huygens Absorbing Boundary Conditions for Electromagnetics. J. Comput. Phys., 226, 354-378, Apr 2007.

[10] T. Hemmi, F. Costen, S. Garcia, R. Himeno, H. Yokota, and M. Mustafa. Efficient Parallel LOD-FDTD Method for Debye-Dispersive Media. IEEE Trans. Antennas Propag., vol. 62, no. 3, pp. 1330-1338, 2014.

[11] W. H. Hayt and Jr. J. A. Buck. Engineering Electromagnetics. McGrawHill College, Singapore, 2000.

[12] J. B. Schneider. Plane Waves in FDTD Simulations and a Nearly Perfect Total-Field/Scattered-Field Boundary. IEEE Trans. Antennas Propag., vol. 52, no. 12, pp. 3280-3287, 2004. 